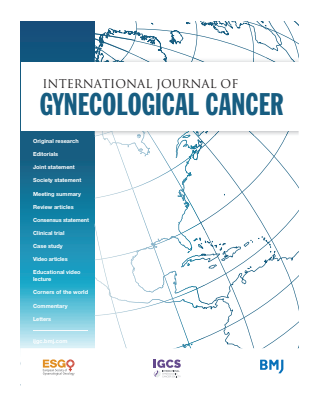

- Additional material is published online only. To view please visit the journal online (http://dx.doi.org/10.1136/ijgc2020-001646).

For numbered affiliations see end of article.

Correspondence to Dr John K Chan, Division of Gynecologic Oncology, Palo Alto Medical Foundation, California Pacific Medical Center, Sutter Health, San Francisco, CA 94109, USA; chanjohn@ sutterhealth.org

Received 21 May 2020 Revised 8 July 2020 Accepted 10 July 2020 Published Online First 4 August 2020

\section{Check for updates}

(C) IGCS and ESGO 2020. № commercial re-use. See rights and permissions. Published by BMJ.

To cite: Abel MK, Chan JK, Chow S, et al. Int J Gynecol Cancer 2020;30:1347-1355.

\title{
Trends and survival outcomes of robotic, laparoscopic, and open surgery for stage II uterine cancer
}

Mary Kathryn Abel, ${ }^{1}$ John K Chan, ${ }^{2}$ Stephanie Chow, ${ }^{3}$ Kathleen Darcy, ${ }^{4,5}$ Chunqiao Tian, ${ }^{4,5}$ Daniel S Kapp, ${ }^{6}$ Amandeep K Mann, ${ }^{7}$ Cheng-I Liao ${ }^{8}$

\section{HIGHLIGHTS}

- Women with stage II uterine cancer have similar 5-year survival after minimally invasive and open hysterectomy

- The use of minimally invasive surgery for stage II uterine cancer increased between 2010 and 2015

- Black women with stage II uterine cancer have more open hysterectomies and worse 5-year survival

\begin{abstract}
Introduction A recent randomized clinical trial showed that minimally invasive surgery led to poorer survival compared with open surgery in early stage cervical cancer. We determined the trends in adoption of minimally invasive surgery and 5-year overall survival outcomes after open, laparoscopic-assisted, and robotic-assisted hysterectomy for stage II uterine cancer with cervical stromal involvement.
\end{abstract}

Methods Data for patients with stage II uterine cancer were acquired from the National Cancer Database from 2010 to 2015. $\chi^{2}$ testing, Kaplan-Meier methods, and Cox models were used for statistical analyses.

Results of 2949 patients, $44.3 \%$ underwent open hysterectomy, $13.9 \%$ underwent laparoscopic hysterectomy, and $41.8 \%$ underwent robotic hysterectomy. The proportion of robotic cases increased from $26.8 \%$ in 2010 to $48.3 \%$ in 2015 (annual percent change 10.1\%), with a decrease in open hysterectomy from $63.3 \%$ to $34.3 \%$ (annual percent change -12.5\%). The overall 5year survival was $77.6 \%$ in robotic, $76.8 \%$ in laparoscopic, and $72.5 \%$ in open hysterectomy $(p=0.045)$; however, after adjusting for known prognostic factors, robotic (HR $1.00,95 \% \mathrm{Cl} 0.82$ to $1.21 ; \mathrm{p}=0.97)$ and laparoscopic hysterectomy (HR $1.09,95 \% \mathrm{Cl} 0.83$ to $1.44 ; \mathrm{p}=0.54$ ) did not portend for improved survival compared with open hysterectomy. Black women (HR 1.59, 95\% Cl 1.25 to 2.02; $\mathrm{p}<0.001$ ) and individuals with co-morbidities (HR 1.45, $95 \% \mathrm{Cl} 1.21$ to $1.75, \mathrm{p}<0.001$ ) had worse adjusted survival and the highest rates of open hysterectomy.

Conclusion The use of minimally invasive surgery for stage II uterine cancer has increased over time, with comparable adjusted 5-year survival after robotic or laparoscopic hysterectomy compared with open hysterectomy. Black women and those with co-morbidities had lowest rates of minimally invasive surgery and the poorest adjusted survival.

\section{INTRODUCTION}

Uterine cancer is the most common gynecologic malignancy in the USA, with an estimated 66650 new cases and 12590 deaths projected in $2020{ }^{1}$
Recommended surgical management includes a total hysterectomy, bilateral salpingectomy-oophorectomy, and lymphadenectomy for endometrioid histological subtypes and more extensive surgery, including a partial omentectomy, for serous and clear cell subtypes. $^{2}$ There has been increased adoption of minimally invasive surgery through a laparoscopic approach, primarily fueled by shorter recovery, lower wound complications, and improved quality of life. ${ }^{34}$

Two randomized clinical trials have found that the use of laparoscopic hysterectomy results in equivalent overall survival to open hysterectomy in patients with early stage uterine cancer. ${ }^{35}$ However, neither of these studies included the use of robotic surgery, a method of minimally invasive surgery that has been widely adopted across the USA and is used at a rate of $25 \%$ at high-volume centers for uterine cancer. ${ }^{6}$ Moreover, these studies excluded patients with stage II uterine cancer with cervical stromal involvement, which has a higher risk of recurrence than stage I disease. ${ }^{7}$ This issue is particularly pertinent given a recent randomized controlled trial showing that minimally invasive surgery was associated with poorer survival compared with open surgery in patients with early stage cervical cancer. ${ }^{8}$ Given the concerns regarding the increased risk of death associated with minimally invasive surgery in early stage cervical cancer, there may exist a similar underlying risk in the use of minimally invasive surgery in stage II uterine cancer with cervical involvement. Thus, the safety of minimally invasive surgery, and particularly robotic surgery, warrants further investigation.

In addition to survival outcomes, trends and disparities in the adoption of minimally invasive surgery for the treatment of uterine cancer is an area of active investigation. Current research on the use of minimally invasive surgery in the treatment of uterine cancer has either not been stratified by stage of disease or has predominantly included patients with stage I uterine cancer. ${ }^{6910}$ Whether the trends seen in 


\section{Original research}

stage I uterine cancer are reflected in stage II disease is unknown and requires further research, particularly because randomized controlled trials are not feasible to perform given the relatively low incidence of stage II uterine cancer. ${ }^{10-12}$

Using the National Cancer Database, we evaluated the 5-year overall survival after open, laparoscopic-assisted, and roboticassisted hysterectomy in a large cohort of patients with locally advanced stage II uterine cancer. In addition, we assessed the trends of surgery in stage II uterine cancer based on race, ethnicity, insurance status, and other socio-economic factors to evaluate whether disparities exist in this study population.

\section{METHODS}

\section{Data source}

The National Cancer Database was established in 1989 and is a nationwide, facility-based, comprehensive clinical surveillance resource and oncology dataset. The data represent more than $70 \%$ of newly diagnosed cancer cases in the USA and include information on patient demographics, clinical characteristics, treatment, and outcomes. ${ }^{1314}$ National Cancer Database Participant User Files from 2010 to 2015 were abstracted in this analysis. Because the data used in this study are derived from a de-identified National Cancer Database file, no institutional review board approval was required.

\section{Cohort selection and characteristics}

Our cohort consisted of patients diagnosed with stage II uterine cancer between 2010 and 2015 who underwent simple or radical hysterectomy. Disease was categorized based on the International Classification of Diseases for Oncology 3rd edition, and the stage of disease was based on the American Joint Committee on Cancer 7th edition. We excluded individuals who did not undergo a pelvic and para-aortic lymphadenectomy, those who were missing surgical approach or lymphadenectomy information, and those who received neoadjuvant chemotherapy or pre-operative radiation therapy.

Patient characteristics abstracted from the database included age at diagnosis, race, insurance, educational attainment of individual's area of residence (defined as percent of adults age 25 or older in the individual's zip code who did not graduate from high school), Charlson-Deyo Comorbidity Index Score, and region of the USA. Healthcare facility type was designated based on the Commission on Cancer and included community cancer programs (100-500 newly diagnosed cancer cases each year with full range of diagnostic and treatment services), comprehensive community cancer programs (more than 500 newly diagnosed cancer cases each year with full range of diagnostic and treatment services), Academic/ Research Programs (participates in postgraduate medical education), and integrated network cancer programs (system of facilities that provide integrated cancer care and is characterized by a unified cancer committee). Clinical characteristics included histological cell type, tumor grade, tumor size, lymphovascular invasion, surgical margin, and adjuvant treatment. Type of surgery was categorized as either open, laparoscopic-assisted, or robotic-assisted. Type of surgery was maintained as laparoscopic or robotic even when conversion to an open approach occurred, as has been referenced in prior literature. ${ }^{15}$

\section{Statistical analysis}

We compared baseline patient characteristics between the surgical approaches using $\chi^{2}$ and ANOVA tests to examine the relationship between categorial and continuous variables, respectively. Annual percent change was used to measure the trend in surgical approaches over time using the Joinpoint Regression Program (Version 4.6.0.0) of the National Cancer Institute. ${ }^{16}$ Kaplan-Meier methods were used for univariate analysis, and the log-rank test was used to make comparisons of overall survival based on treatment modality. Multivariate analyses with hazard ratios (HRs) and 95\% confidence intervals (Cls) were estimated using Cox proportional hazards regression to assess the relationship of surgical treatment modality and 5-year overall survival after adjusting for important demographic, clinico-pathologic, and treatment factors. Statistical analyses were performed with SPSS software (Version 26).

\section{RESULTS}

\section{Clinical and demographic patient characteristics}

Between 2010 and 2015, 2949 patients were diagnosed with stage II uterine cancer and received open, laparoscopic, or robotic hysterectomy with pelvic and para-aortic lymphadenectomy. The demographic and clinico-pathologic characteristics of the study population are shown in Table 1. The median age of the cohort was 64 years (range 25-90). Whites, Blacks, Hispanic, Asian/Pacific Islanders, and other races comprised $75.4 \%, 13.0 \%, 7.1 \%, 3.0 \%$, and $1.6 \%$ of the population, respectively. Overall, $22.8 \%$ of patients resided in the Northeast, while $29.7 \%, 32.3 \%$, and $15.2 \%$ resided in the Midwest, South, and West, respectively. Ninety three percent of patients had insurance and $26.6 \%$ had a Charlson-Deyo Comorbidity Index Score of $\geq 1$.

\section{Trends by route of hysterectomy}

The majority of patients underwent either open (44.3\%) or robotic hysterectomy (41.8\%), and the remainder underwent laparoscopic hysterectomy (13.9\%, Table 1). We analyzed the changes in surgical method across the study timeframe. The proportion of robotic hysterectomies increased from $26.8 \%$ in 2010 to $48.3 \%$ in 2015 , with an annual percent change of $10.1 \%$ ( $p<0.05$, Figure 1$)$. There was a corresponding decrease in open hysterectomy from $63.3 \%$ to $34.3 \%$ (annual percent change $-12.5 \%, p<0.05$ ), while laparoscopic hysterectomy showed an increase from $9.9 \%$ to $17.4 \%$ (annual percent change 11.2\%).

White individuals were more likely to undergo robotic hysterectomy (44.4\%) than black (31.4\%), Hispanic (36.2\%), and Asian/ Pacific Islander individuals $(33.3 \%, p<0.001$; Table 1 , online supplementary figure 1). Black women had the highest rates of open hysterectomy at $54.2 \%$, while Hispanic women had the highest rates of laparoscopic hysterectomy at $19.5 \%$. Patients seen in integrated network cancer programs had the highest rate of robotic hysterectomy (45.2\%), and those receiving care in community cancer programs had the highest rate of open hysterectomy (56.1\%; $p=0.043)$. Individuals with insurance were also more likely to undergo robotic hysterectomy than those who were uninsured $(42.8 \%$ vs $28.8 \% ; p=0.004)$. Additionally, those with a higher rate of educational attainment in their area of residence (no high school degree $<13 \%$ (44.8\%) vs $>13 \%(36.7 \%) ; p<0.001)$ and those with 
Table 1 Patient demographics by route of hysterectomy

\begin{tabular}{|c|c|c|c|c|c|}
\hline & \multirow{2}{*}{$\begin{array}{l}\text { Open } \\
\mathbf{n}(\%) \\
\end{array}$} & \multirow{2}{*}{$\begin{array}{l}\text { Laparoscopic } \\
\text { n (\%) }\end{array}$} & \multirow{2}{*}{$\begin{array}{l}\text { Robotic } \\
\text { n (\%) }\end{array}$} & \multirow{2}{*}{$\begin{array}{c}\text { Total } \\
\mathbf{n}(\%)\end{array}$} & \multirow[b]{2}{*}{$P$ value } \\
\hline & & & & & \\
\hline No of cases & 1305 (44.3) & $410(13.9)$ & $1234(41.8)$ & $2949(100)$ & \\
\hline Age, years & & & & & $0.891^{*}$ \\
\hline Mean \pm SD & $63.17 \pm 11.30$ & $63.21 \pm 11.01$ & $63.34 \pm 10.71$ & $63.25 \pm 11.01$ & \\
\hline Median & 63 & 64 & 64 & 64 & \\
\hline Min-Max & $25-90$ & $28-90$ & $31-90$ & $25-90$ & \\
\hline Race & & & & & $<0.001$ \\
\hline White & $947(42.6)$ & $289(13.0)$ & $987(44.4)$ & $2223(100)$ & \\
\hline Black & $207(54.2)$ & $55(14.4)$ & $120(31.4)$ & $382(100)$ & \\
\hline Hispanic & $93(44.3)$ & $41(19.5)$ & $76(36.2)$ & $210(100)$ & \\
\hline Asian/Pacific Islander & $43(49.4)$ & $15(17.2)$ & $29(33.3)$ & $87(100)$ & \\
\hline Other/unknown & 15 (31.9) & $10(21.3)$ & $22(46.8)$ & $47(100)$ & \\
\hline Region of the USA & & & & & $<0.001$ \\
\hline Northeast & $310(46.1)$ & $96(14.3)$ & $266(39.6)$ & $672(100)$ & \\
\hline Midwest & $387(47.0)$ & $70(8.5)$ & $367(44.5)$ & $824(100)$ & \\
\hline South & $411(43.2)$ & $145(15.2)$ & $396(41.6)$ & $952(100)$ & \\
\hline West & $173(38.5)$ & $91(20.3)$ & $185(41.2)$ & 449 (100) & \\
\hline Unknown & $24(46.2)$ & $8(15.4)$ & $20(38.5)$ & $52(100)$ & \\
\hline Healthcare facility type & & & & & 0.043 \\
\hline Community cancer program & $46(56.1)$ & $8(9.8)$ & $28(34.1)$ & $82(100)$ & \\
\hline Comprehensive community cancer program & $429(41.5)$ & $159(15.4)$ & $445(43.1)$ & $1033(100)$ & \\
\hline Academic/research program & $613(46.1)$ & $181(13.6)$ & $537(40.3)$ & $1331(100)$ & \\
\hline Integrated network cancer program & $193(42.8)$ & $54(12.0)$ & $204(45.2)$ & $451(100)$ & \\
\hline Insurance status & & & & & 0.004 \\
\hline No & $90(55.2)$ & $26(16.0)$ & $47(28.8)$ & $163(100)$ & \\
\hline Yes & $1195(43.4)$ & $380(13.8)$ & $1177(42.8)$ & $2752(100)$ & \\
\hline Unknown & $20(58.8)$ & $4(11.8)$ & $10(29.4)$ & $34(100)$ & \\
\hline No high school degree 2008-2012, \% & & & & & $<0.001$ \\
\hline$\geq 13 \%$ & 547 (49.2) & $157(14.1)$ & $408(36.7)$ & 1112 (100) & \\
\hline$<13 \%$ & $724(41.5)$ & $239(13.7)$ & $782(44.8)$ & $1745(100)$ & \\
\hline Charlson-Deyo Comorbidity Index Score & & & & & 0.001 \\
\hline 0 & $922(42.6)$ & $293(13.5)$ & $949(43.9)$ & $2164(100)$ & \\
\hline$\geq 1$ & $383(48.8)$ & $117(14.9)$ & $285(36.3)$ & $785(100)$ & \\
\hline
\end{tabular}

Data are $\mathrm{n}(\%)$ unless otherwise specified.

Percentages are in row percent, and proportions were compared using $\chi^{2}$ tests.

*A t-test was used to calculate $p$ values for the mean difference of age (years) for open vs laparoscopic vs robotic surgery.

no co-morbidities $(43.9 \%$ for Charlson-Deyo Comorbidity Index Score 0 vs $36.3 \%$ for individuals with Charlson-Deyo Comorbidity Index Score $1 ; \mathrm{p}=0.001$ ) were also more likely to undergo robotic hysterectomy. Compared with white patients, black patients were more likely to have more co-morbidities (34.6\% vs $24.2 \%$; $p<0.001)$, lack insurance $(8.1 \%$ vs $3.6 \%)$, and receive care at academic/research programs $(54.0 \%$ vs $44.3 \% ; p<0.001)$ or integrated network cancer programs ( $17.6 \%$ vs $15.7 \% ; p<0.001)$.

Mean tumor size was $6.20 \mathrm{~cm}$ with open hysterectomy, $5.82 \mathrm{~cm}$ in laparoscopic hysterectomy, and $5.69 \mathrm{~cm}$ in robotic hysterectomy $(p<0.001$, Table 2). Individuals with endometrioid carcinoma were more likely to undergo robotic hysterectomy (44.2\%) than those with non-endometrioid carcinoma sub-types (serous carcinoma: $41.1 \%$, clear cell carcinoma: $32.7 \%$, carcinosarcoma: $27.6 \%$; $p<0.001$ ). This trend was not as pronounced for laparoscopic hysterectomy (endometrioid carcinoma: $14.4 \%$, serous carcinoma: $14.8 \%$, clear cell carcinoma: $9.6 \%$; carcinosarcoma: $10.0 \%$ ). Patients with poorly differentiated tumors had the highest rates of open hysterectomy $(51.1 \%)$, while laparoscopic and robotic approaches were used most frequently for moderately differentiated tumors (15.6\%) and well differentiated tumors $(47.7 \%)$, respectively $(p<0.001)$. Laparoscopic hysterectomy was performed most frequently for $<2 \mathrm{~cm}$ 


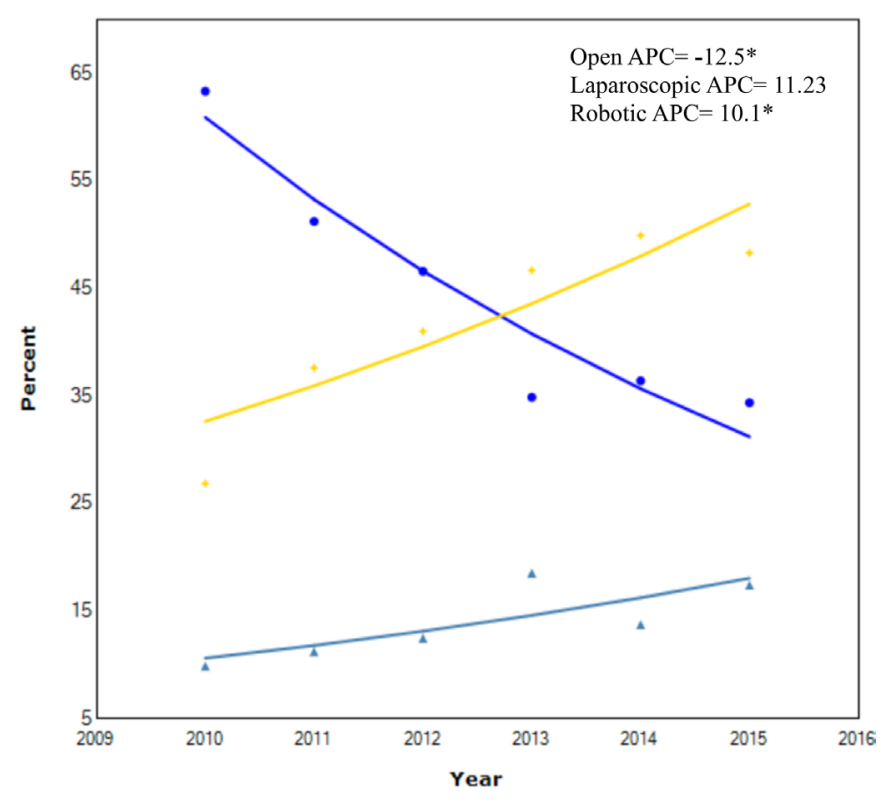

$\begin{array}{lcccccc}\quad \text { Year } & \mathbf{2 0 1 0} & \mathbf{2 0 1 1} & \mathbf{2 0 1 2} & \mathbf{2 0 1 3} & \mathbf{2 0 1 4} & \mathbf{2 0 1 5} \\ \text { Open } & 63.3 \% & 51.2 \% & 46.6 \% & 34.8 \% & 36.4 \% & 34.3 \% \\ \text { Laparoscopic } & 9.9 \% & 11.2 \% & 12.4 \% & 18.5 \% & 13.7 \% & 17.4 \% \\ \text { Robotic } & 26.8 \% & 37.6 \% & 41.0 \% & 46.7 \% & 49.9 \% & 48.3 \%\end{array}$

Figure 1 Trends by route of hysterectomy from 2010 to 2015 in the USA. * indicates that the annual percent change (APC) is significantly different from zero at the alpha $=0.05$ level.

while robotic hysterectomy was used for intermediate tumors of $2-4 \mathrm{~cm}(48.9 \%, p<0.001)$.

\section{Survival analysis and treatment}

The 5-year survival rate by surgical method is shown in Figure 2. The unadjusted 5 -year survival rate was highest for robotic hysterectomy $(77.6 \%)$, followed by laparoscopic hysterectomy $(76.8 \%)$ and open hysterectomy $(72.5 \% ; p=0.045)$. To evaluate the effect of demographic factors and route of hysterectomy on 5-year survival, we used a Cox proportional hazards regression model that included important demographic, clinico-pathologic, and treatment factors. After adjusting for these factors, there was no association between survival and surgical method (laparoscopic vs open: HR 1.09, $95 \% \mathrm{Cl} 0.83$ to 1.44 ; $\mathrm{p}=0.54$; robotic vs open: $\mathrm{HR} 1.00,95 \% \mathrm{Cl} 0.82$ to $1.21 ; p=0.97$, Table 3 ).

Black compared with white race ( $\mathrm{HR} 1.59,95 \% \mathrm{Cl} 1.25$ to 2.02; $\mathrm{p}<0.001)$ and having a Charlson-Deyo Comorbidity Index Score of 1 versus 0 (HR $1.45,95 \% \mathrm{Cl} 1.21$ to $1.75 ; p<0.001$ ) were associated with worse adjusted 5 -year overall survival (Table 3). Additionally, individuals with carcinosarcoma compared with endometrial carcinoma (HR 1.70, 95\% Cl 1.29 to 2.24; $p<0.001$ ) and those with lymphovascular invasion ( $\mathrm{HR} 1.71,95 \% \mathrm{Cl} 1.42$ to 2.06 ; $\mathrm{p}<0.001$ ) had worse survival. Receiving either adjuvant radiation therapy alone (HR $0.69,95 \% \mathrm{Cl} 0.54$ to $0.87 ; \mathrm{p}=0.002$ ) or adjuvant radiation therapy and chemotherapy ( $\mathrm{HR} 0.63,95 \% \mathrm{Cl} 0.48$ to 0.82 , $\mathrm{p}=0.001$ ) resulted in improved 5-year survival compared with those who did not receive adjuvant therapy. Surgical field, tumor size, and surgical margin status were not associated with improved or worsened survival.

\section{DISCUSSION}

Uterine cancer is the most common pelvic gynecologic malignancy in the USA, with a rising incidence and an increase in mortality rate of $1.1 \%$ per year over the past two decades. ${ }^{17}$ Although prior studies have evaluated the management of stage II uterine cancer with cervical stromal involvement, there is currently limited literature that evaluates the effect of minimally invasive surgical techniques on survival outcomes in stage II uterine cancer, which is particularly important given recent literature suggesting reduced survival with the use of minimally invasive surgery in the treatment of early stage cervical cancer. ${ }^{8}{ }^{18-20}$ In our retrospective cohort study of 2949 patients, we found that minimally invasive approaches including laparoscopic and robotic hysterectomy appear to have comparable adjusted 5-year survival outcomes to open hysterectomy for stage II uterine cancer. Moreover, individuals who are often underserved by our healthcare system, including black and uninsured patients, have limited access to minimally invasive surgery for the treatment of stage II uterine cancer.

In two randomized controlled trials, minimally invasive approaches were found to have equivalent survival outcomes to an open approach for early stage uterine cancer. ${ }^{3-5}$ Walker et al found no difference in disease-free or overall survival for patients undergoing laparoscopy versus open hysterectomy for stage I or IIA uterine cancer. ${ }^{45}$ Moreover, Janda et al randomized patients with stage I disease to receive laparoscopic or open hysterectomy and found no difference in recurrence rates or overall survival. ${ }^{3}$ However, patients with stage II disease with cervical involvement were excluded from these trials. ${ }^{7}$ As such, the safety and efficacy of minimally invasive surgery in this population has not been well studied.

Surgical route has been studied in the setting of cervical cancer and may shed light on the most appropriate surgical treatment for uterine cancer with cervical involvement. While retrospective studies have found no difference in the rate of recurrence or death following robotic or laparoscopic hysterectomy compared with open hysterectomy for cervical cancer, ${ }^{81-23}$ a recent pivotal prospective randomized controlled trial of 319 women with early stage cervical cancer found that minimally invasive surgery, which included both laparoscopic and robotic approaches, had a lower rate of diseasefree survival and overall survival than open abdominal radical hysterectomy. ${ }^{8}$ In this current analysis of stage II uterine cancer with cervical involvement, our results did not show decreased survival associated with minimally invasive surgery. There are a number of reasons why this might be the case. It is possible that the location of the tumor in uterine cancer generally remains contained in the corpus while the majority of cervical cancer tumors are exophytic and exposed to the vaginal cavity. When a surgeon enters the vagina with a minimally invasive approach, the insufflation of carbon dioxide gas may facilitate local spread of cervical cancer from the enclosed vaginal cavity into the peritoneum. In contrast, in most uterine cancers the ectocervix remains uninvolved and may contain the tumor within the uterine cavity, thereby minimizing the risk of spread during minimally invasive surgery. The effect of insufflation on tumor cell growth has also been discussed in prior studies; however, the cervical involvement in stage II uterine cancer is not in direct contact with the gas and may not promote tumor growth. ${ }^{24} 25$ 
Table 2 Tumor characteristics by route of hysterectomy

\begin{tabular}{|c|c|c|c|c|c|}
\hline & Open & Laparoscopic & Robotic & Total & \\
\hline & n (\%) & n (\%) & n (\%) & n (\%) & $P$ value \\
\hline No of cases & $1305(44.3)$ & $410(13.9)$ & $1234(41.8)$ & $2949(100)$ & \\
\hline Surgical field & & & & & $<0.001$ \\
\hline Simple hysterectomy & $1100(42.5)$ & $373(14.4)$ & $1115(43.1)$ & $2588(100)$ & \\
\hline Radical hysterectomy & $205(56.8)$ & $37(10.2)$ & $119(33.0)$ & $361(100)$ & \\
\hline Histology & & & & & $<0.001$ \\
\hline Endometrioid carcinoma & 907 (41.3) & $317(14.4)$ & $970(44.2)$ & $2194(100)$ & \\
\hline Serous carcinoma & $164(44.1)$ & $55(14.8)$ & $153(41.1)$ & $372(100)$ & \\
\hline Clear cell carcinoma & $60(57.7)$ & $10(9.6)$ & $34(32.7)$ & $104(100)$ & \\
\hline Carcinosarcoma & $174(62.4)$ & $28(10.0)$ & $77(27.6)$ & $279(100)$ & \\
\hline Grade & & & & & $<0.001$ \\
\hline Well differentiated & $186(37.6)$ & $73(14.7)$ & $236(47.7)$ & $495(100)$ & \\
\hline Moderately differentiated & $339(40.9)$ & $129(15.6)$ & $360(43.5)$ & $828(100)$ & \\
\hline Poorly differentiated & $627(51.1)$ & $156(12.7)$ & $443(36.1)$ & $1226(100)$ & \\
\hline Unknown & $153(38.3)$ & $52(13.0)$ & $195(48.8)$ & $400(100)$ & \\
\hline \multicolumn{6}{|l|}{ Tumor size (cm) } \\
\hline Mean \pm SD & $6.20 \pm 5.28$ & $5.82 \pm 1.02$ & $5.10 \pm 13.41$ & $5.69 \pm 4.80$ & $<0.001^{*}$ \\
\hline Tumor size stratified (cm) & & & & & $<0.001$ \\
\hline$<2$ & $90(38.3)$ & $41(17.4)$ & $104(44.2)$ & $235(100)$ & \\
\hline$\geq 2$ and $<4$ & $259(36.6)$ & $103(14.5)$ & $346(48.9)$ & $708(100)$ & \\
\hline$\geq 4$ & $767(48.1)$ & $211(13.2)$ & $617(38.7)$ & $1595(100)$ & \\
\hline Unknown & $189(46.0)$ & $55(13.4)$ & $167(40.6)$ & $411(100)$ & \\
\hline Lymphovascular invasion & & & & & 0.258 \\
\hline No & $799(44.9)$ & $227(12.7)$ & $755(42.4)$ & $1781(100)$ & \\
\hline Yes & $409(43.1)$ & $148(15.6)$ & $393(41.4)$ & $950(100)$ & \\
\hline Unknown & $97(44.5)$ & $35(16.1)$ & $86(39.4)$ & $218(100)$ & \\
\hline Surgical margin & & & & & 0.518 \\
\hline Negative & 1192 (44.2) & $376(13.9)$ & $1128(41.8)$ & $2696(100)$ & \\
\hline Positive & $30(42.3)$ & $6(8.5)$ & $35(49.3)$ & 71 (100) & \\
\hline Unknown & $83(45.6)$ & $28(15.4)$ & $71(39.0)$ & $182(100)$ & \\
\hline Adjuvant treatment & & & & & 0.001 \\
\hline No & $341(47.6)$ & $97(13.5)$ & 279 (38.9) & $717(100)$ & \\
\hline Chemotherapy only & $142(50.9)$ & $35(12.5)$ & $102(36.6)$ & $279(100)$ & \\
\hline Radiation therapy only & $475(39.5)$ & $171(14.2)$ & $556(46.3)$ & $1202(100)$ & \\
\hline Radiation therapy and chemotherapy & $297(47.1)$ & $83(13.2)$ & $251(39.8)$ & $631(100)$ & \\
\hline Unknown & $50(41.7)$ & $24(20.0)$ & 46 (38.3) & $120(100)$ & \\
\hline
\end{tabular}

Data are $\mathrm{n}(\%)$ unless otherwise specified.

Percentages are in row percent, and proportions were compared using $\chi^{2}$ tests.

${ }^{*} A$ t-test was used to calculate $p$ values for the mean difference of tumor size $(\mathrm{cm})$ for open vs laparoscopic vs robotic surgery.

Although we found that minimally invasive approaches have comparable survival outcomes to open hysterectomy in our retrospective analysis, it is important to note that individuals who are marginalized by the healthcare system did not have the same rates of minimally invasive surgery. For example, black women had higher rates of open hysterectomy compared with laparoscopic and robotic hysterectomy, and black race remained an independent predictor for poorer survival even after adjusting for surgery type, grade, histology, and other socio-demographic, clinico-pathologic, and treatment factors. This is consistent with prior studies that have shown limited access to minimally invasive techniques and worse outcomes in black women with uterine cancer, cervical cancer, and benign conditions like fibroids and prolapse. ${ }^{6} 12$ 26-28 However, it is important to note that this relationship is complex and 


\section{Original research}

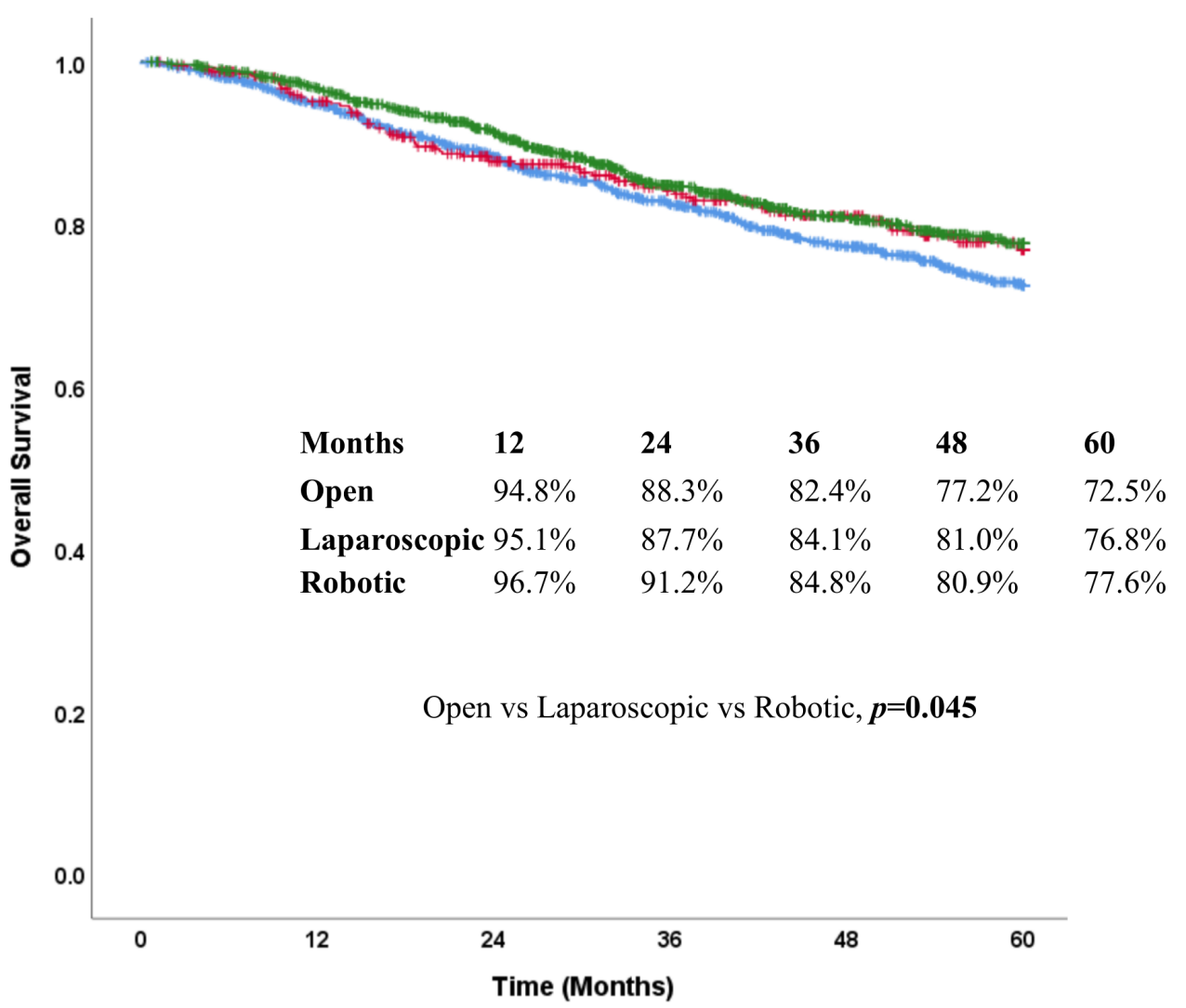

Figure 2 Five-year unadjusted survival estimates by route of hysterectomy.

likely dependent on other factors that are not accounted for in large databases, including the effect of implicit bias, healthcare literacy, and historical racism in the healthcare system. Additionally, individuals without insurance were less likely to receive robotic hysterectomy than laparoscopic and open hysterectomy. As noted in a paper by Jacoby et al, for women undergoing hysterectomy without private insurance relying on either 'no charge/charity' or self-pay options, there may be an incentive to avoid laparoscopy or robotic surgery to limit cost to the hospital or patient. ${ }^{12}$ Further studies that incorporate both the molecular characteristics of these tumors and socio-economic and historical factors associated with survival are necessary to fully understand these disparities. ${ }^{29-31}$

This study was limited due to the retrospective nature of the research, which precludes us from making definitive treatment recommendations. Ultimately, randomized controlled trials are needed to thoroughly evaluate the effect of surgical approach on survival outcomes for patients with stage II uterine cancer with cervical stromal involvement. However, given the limited number of stage II uterine cancer diagnoses in the USA, this may prove challenging. For example, even after combining patients with stages I and IIA uterine cancer, the LAP2 trial required nearly a decade to enroll 2616 patients. ${ }^{4}$ Importantly, given the limitations of the dataset, we were unable to determine whether the diagnosis of stage II uterine cancer was made pre-operatively or post-operatively, which could have influenced the surgeon's decision to use a minimally invasive surgery versus open approach with a simple or radical hysterectomy. Additionally, physician skill set, years in practice, access to robotic surgical devices, physician rationale, and prior training, including training in minimally invasive surgical techniques, may have influenced the choice of surgical method, and future studies are needed to evaluate this impact. We did not adjust for parametrial involvement of uterine cancer in our study. Finally, due to the limitations of our database, we were only able to analyze overall survival rather than progression-free survival or disease-specific survival. For example, we know that individuals with uterine cancer are most likely to die from cardiovascular disease, not uterine cancer; however, we were unable to assess this effect in our population. ${ }^{32}{ }^{33}$ Despite these limitations, our study contributes to the current understanding of the effect of surgical approach on survival outcomes in stage II uterine cancer, particularly due to the large sample size, national representation, and inclusion of the robotic approach in minimally invasive techniques. More studies on long-term outcomes of minimally invasive surgery for stage II uterine cancer, including length of hospital stay, post-operative complications, re-admission rates, and overall economic impact on the healthcare system, are needed to appreciate the larger implications of choice of surgical method in the treatment of this disease.

\section{CONCLUSION}

The choice of route of hysterectomy for the treatment of stage II uterine cancer with cervical involvement should be based on a number of factors, including patient and provider preference and the unique clinical presentation. However, as the rate of minimally invasive surgery increases, it is important to evaluate the possible disparities and differences in survival outcomes from the utilization of this technique. Our study shows that minimally invasive hysterectomy has comparable 5-year survival outcomes to open hysterectomy for stage II uterine cancer; moreover, we found that minimally invasive surgery is used less commonly in black patients and those with co-morbidities. 


\begin{tabular}{|c|c|c|c|}
\hline Factors & HR & $95 \% \mathrm{Cl}$ & $P$ value \\
\hline \multicolumn{4}{|l|}{ Surgical method } \\
\hline Open & Ref & & \\
\hline Laparoscopic & 1.09 & 0.83 to 1.44 & 0.538 \\
\hline Robotic & 1.00 & 0.82 to 1.21 & 0.970 \\
\hline \multicolumn{4}{|l|}{ Facility type } \\
\hline Community cancer program & Ref & & \\
\hline Comprehensive community cancer program & 1.00 & 0.57 to 1.75 & 0.996 \\
\hline Academic/research program & 0.97 & 0.55 to 1.70 & 0.906 \\
\hline Integrated network cancer program & 0.81 & 0.45 to 1.46 & 0.485 \\
\hline \multicolumn{4}{|l|}{ Region } \\
\hline Northeast & Ref & & \\
\hline Midwest & 1.21 & 0.95 to 1.53 & 0.118 \\
\hline South & 0.86 & 0.67 to 1.10 & 0.234 \\
\hline West & 1.07 & 0.79 to 1.45 & 0.676 \\
\hline \multicolumn{4}{|l|}{ Race } \\
\hline White & Ref & & \\
\hline Black & 1.59 & 1.25 to 2.02 & 0.000 \\
\hline Hispanic & 0.86 & 0.55 to 1.32 & 0.481 \\
\hline Asian/Pacific Islander & 1.15 & 0.65 to 2.02 & 0.629 \\
\hline Other/unknown & 1.00 & 0.47 to 2.11 & 0.991 \\
\hline \multicolumn{4}{|l|}{ Insurance or not } \\
\hline No & Ref & & \\
\hline Yes & 0.99 & 0.60 to 1.63 & 0.966 \\
\hline Unknown & 0.80 & 0.26 to 2.43 & 0.694 \\
\hline \multicolumn{4}{|l|}{ No high school degree 2008-2012, \% } \\
\hline$\geq 13 \%$ & Ref & & \\
\hline$<13 \%$ & 1.10 & 0.90 to 1.33 & 0.352 \\
\hline \multicolumn{4}{|l|}{ Charlson--Deyo Comorbidity Index Score } \\
\hline 0 & Ref & & \\
\hline$\geq 1$ & 1.45 & 1.21 to 1.75 & 0.000 \\
\hline \multicolumn{4}{|l|}{ Surgical field } \\
\hline Simple hysterectomy & Ref & & \\
\hline Radical hysterectomy & 0.92 & 0.70 to 1.22 & 0.567 \\
\hline \multicolumn{4}{|l|}{ Histology } \\
\hline Endometrioid carcinoma & Ref & & \\
\hline Serous carcinoma & 0.89 & 0.66 to 1.20 & 0.442 \\
\hline Clear cell carcinoma & 0.71 & 0.43 to 1.17 & 0.180 \\
\hline Carcinosarcoma & 1.70 & 1.29 to 2.24 & 0.000 \\
\hline \multicolumn{4}{|l|}{ Grade } \\
\hline Well differentiated & Ref & & \\
\hline Moderately differentiated & 1.50 & 1.03 to 2.20 & 0.037 \\
\hline Poorly differentiated & 3.12 & 2.13 to 4.57 & 0.000 \\
\hline Unknown & 1.58 & 1.02 to 2.45 & 0.041 \\
\hline \multicolumn{4}{|l|}{ Tumor size (cm) } \\
\hline$<2$ & Ref & & \\
\hline$\geq 2$ and $<4$ & 0.94 & 0.63 to 1.39 & 0.754 \\
\hline
\end{tabular}




\section{Original research}

\begin{tabular}{|c|c|c|c|}
\hline$\geq 4$ & 1.17 & 0.80 to 1.69 & 0.418 \\
\hline Unknown & 1.23 & 0.81 to 1.87 & 0.326 \\
\hline \multicolumn{4}{|l|}{ Lymphovascular invasion } \\
\hline No & Ref & & \\
\hline Unknown & 0.88 & 0.60 to 1.30 & 0.528 \\
\hline \multicolumn{4}{|l|}{ Surgical margin } \\
\hline Negative & Ref & & \\
\hline Positive & 1.27 & 0.78 to 2.07 & 0.343 \\
\hline Chemotherapy only & 0.79 & 0.58 to 1.08 & 0.135 \\
\hline Radiation therapy only & 0.69 & 0.54 to 0.87 & 0.002 \\
\hline Radiation therapy and chemotherapy & 0.63 & 0.48 to 0.82 & 0.001 \\
\hline Unknown & 0.87 & 0.57 to 1.34 & 0.539 \\
\hline
\end{tabular}

\begin{abstract}
Author affiliations
${ }^{1}$ School of Medicine, University of California San Francisco, San Francisco, California, USA

2Division of Gynecologic Oncology, Palo Alto Medical Foundation, California Pacific Medical Center, Sutter Health, San Francisco, California, USA

${ }^{3}$ Department of Obstetrics and Gynecology, Stanford University School of Medicine, Stanford, California, USA

${ }^{4}$ Gynecologic Cancer Center of Excellence, Department of Obstetrics \& Gynecology, Uniformed Services University of the Health Sciences, Walter Reed National Military Medical Center, Bethesda, Maryland, USA

${ }^{5}$ Henry M Jackson Foundation for the Advancement of Military Medicine Inc, Bethesda, Maryland, USA

${ }^{6}$ Department of Radiation Oncology, Stanford University School of Medicine, Palo Alto, California, USA

${ }^{7}$ Division of Gynecologic Oncology, Palo Alto Medical Foundation Research Institute, Palo Alto, California, USA

${ }^{8}$ Department of Obstetrics and Gynecology, Kaohsiung Veterans General Hospital, Kaohsiung, Taiwan
\end{abstract}

Contributors MKA: methodology; writing original draft; writing review and editing; visualization. JKC: conceptualization; writing review and editing; visualization; supervision; project administration; funding acquisition. SC: conceptualization; writing review and editing. KD: writing review and editing. CT: writing review and editing. DSK: conceptualization; writing review and editing. AKM: methodology; writing review and editing. C-IL: methodology; software; formal analysis; investigation; resources; writing review and editing.

Funding We would like to acknowledge The Fisher Family Fund and Denise Cobb Hale for their generous administrative support.

Competing interests None declared.

Patient consent for publication Not required.

Provenance and peer review Not commissioned; externally peer reviewed.

Data availability statement Data may be obtained from a third party and are not publicly available. The data for this study was obtained with permission from the National Cancer Database.

\section{REFERENCES}

1 Siegel RL, Miller KD, Jemal A. Cancer statistics, 2020. CA A Cancer $J$ Clin 2020;70:7-30.
2 Frost JA, Webster KE, Bryant A, et al. Lymphadenectomy for the management of endometrial cancer. Cochrane Database Syst Rev 2017;283.

3 Janda M, Gebski V, Davies LC, et al. Effect of total laparoscopic hysterectomy vs total abdominal hysterectomy on diseasefree survival among women with stage I endometrial cancer: a randomized clinical trial. JAMA 2017;317:1224-33.

4 Walker JL, Piedmonte MR, Spirtos NM, et al. Laparoscopy compared with laparotomy for comprehensive surgical staging of uterine cancer: Gynecologic Oncology Group Study LAP2. J Clin Oncol 2009;27:5331-6.

5 Walker JL, Piedmonte MR, Spirtos NM, et al. Recurrence and survival after random assignment to laparoscopy versus laparotomy for comprehensive surgical staging of uterine cancer: Gynecologic Oncology Group LAP2 Study. J Clin Oncol 2012;30:695-700.

6 Chan JK, Gardner AB, Taylor K, et al. The centralization of robotic surgery in high-volume centers for endometrial cancer patients--a study of 6560 cases in the U.S. Gynecol Oncol 2015;138:128-32.

7 Lewin SN, Herzog TJ, Barrena Medel NI, Medel NIB, et al. Comparative performance of the 2009 International Federation of Gynecology and Obstetrics' staging system for uterine corpus cancer. Obstet Gynecol 2010;116:1141-9.

8 Ramirez PT, Frumovitz M, Pareja R, et al. Minimally invasive versus abdominal radical hysterectomy for cervical cancer. N Engl J Med Overseas Ed 2018;379:1895-904.

9 Yu X, Lum D, Kiet TK, et al. Utilization of and charges for robotic versus laparoscopic versus open surgery for endometrial cancer. $J$ Surg Oncol 2013;107:653-8.

10 Esselen KM, Vitonis A, Einarsson J, et al. Health care disparities in hysterectomy for gynecologic cancers: data from the 2012 national inpatient sample. Obstet Gynecol 2015;126:1029-39.

11 Price JT, Zimmerman LD, Koelper NC, et al. Social determinants of access to minimally invasive hysterectomy: reevaluating the relationship between race and route of hysterectomy for benign disease. Am J Obstet Gynecol 2017;217:572.e1-572.e10.

12 Jacoby VL, Autry A, Jacobson G, et al. Nationwide use of laparoscopic hysterectomy compared with abdominal and vaginal approaches. Obstet Gynecol 2009;114:1041-8.

13 Bilimoria KY, Bentrem DJ, Stewart AK, et al. Comparison of Commission on Cancer-approved and -nonapproved hospitals in the United States: implications for studies that use the National Cancer Data Base. J Clin Oncol 2009;27:4177-81.

14 Klein DA, Mann AK, Freeman AH, et al. Chemotherapy alone for patients 75 years and older with epithelial ovarian cancer -is interval cytoreductive surgery still needed? Am J Obstet Gynecol 2020;222:170.e1-170.e11. 
15 Melamed A, Margul DJ, Chen L, et al. Survival after minimally invasive radical hysterectomy for early-stage cervical cancer. $N$ Engl $J$ Med 2018;379:1905-14.

16 Joinpoint Regression Program. Statistical methodology and applications branch, surveillance research program, National Cancer Institute, 2018.

17 Henley SJ, Miller JW, Dowling NF, et al. Uterine cancer incidence and mortality - United States, 1999-2016. MMWR Morb Mortal Wkly Rep 2018;67:1333-8.

18 Cahan B, Kim JH, Schultheiss TE, et al. Stage I and II endometrial adenocarcinoma: analysis of 2009 FIGO staging revision and impact on survival by adjuvant therapy. Am J Clin Oncol 2018;41:302-6.

19 Orezzoli JP, Sioletic S, Olawaiye A, et al. Stage II endometrioid adenocarcinoma of the endometrium: clinical implications of cervical stromal invasion. Gynecol Oncol 2009;113:316-23.

20 Wright JD, Fiorelli J, Kansler AL, et al. Optimizing the management of stage II endometrial cancer: the role of radical hysterectomy and radiation. Am J Obstet Gynecol 2009;200:419.e1-419.e7.

21 Sert BM, Boggess JF, Ahmad S, et al. Robot-assisted versus open radical hysterectomy: a multi-institutional experience for early-stage cervical cancer. Eur J Surg Oncol 2016;42:513-22.

22 Shah CA, Beck T, Liao JB, et al. Surgical and oncologic outcomes after robotic radical hysterectomy as compared to open radical hysterectomy in the treatment of early cervical cancer. J Gynecol Oncol 2017;28:e82.

23 Wang Y-zhou, Deng L, Xu H-cheng, et al. Laparoscopy versus laparotomy for the management of early stage cervical cancer. BMC Cancer 2015;15:928.

24 Lin F, Pan L, Li L, et al. Effects of a simulated CO2 pneumoperitoneum environment on the proliferation, apoptosis, and metastasis of cervical cancer cells in vitro. Med Sci Monit 2014;20:2497-503.

25 Volz J, Köster S, Spacek Z, et al. The influence of pneumoperitoneum used in laparoscopic surgery on an intraabdominal tumor growth. Cancer 1999;86:770-4.

26 Wu JM, Wechter ME, Geller EJ, et al. Hysterectomy rates in the United States, 2003. Obstet Gynecol 2007;110:1091-5.

27 Abenhaim HA, Azziz R, Hu J, et al. Socioeconomic and racial predictors of undergoing laparoscopic hysterectomy for selected benign diseases: analysis of 341487 hysterectomies. J Minim Invasive Gynecol 2008;15:11-15.

28 Benard VB, Watson M, Saraiya M, et al. Cervical cancer survival in the United States by race and stage (2001-2009): findings from the CONCORD-2 study. Cancer 2017;123 Suppl 24:5119-37.

29 Maxwell GL, Allard J, Gadisetti CVR, et al. Transcript expression in endometrial cancers from black and white patients. Gynecol Oncol 2013;130:169-73.

30 Maxwell GL, Shoji Y, Darcy K, et al. Micrornas in endometrial cancers from black and white patients. Am J Obstet Gynecol 2015;212:e10:e1-191.

31 Bateman NW, Dubil EA, Wang G, et al. Race-specific molecular alterations correlate with differential outcomes for black and white endometrioid endometrial cancer patients. Cancer 2017;123:4004-12.

32 Ward KK, Shah NR, Saenz CC, et al. Cardiovascular disease is the leading cause of death among endometrial cancer patients. Gynecol Oncol 2012;126:176-9.

33 Felix AS, Bower JK, Pfeiffer RM, et al. High cardiovascular disease mortality after endometrial cancer diagnosis: results from the Surveillance, Epidemiology, and End Results (SEER) database. Int J Cancer 2017;140:555-64. 